\title{
Analysis of superframe adjustment and beacon transmission for IEEE 802.15.4 cluster tree networks
}

\author{
Bih-Hwang Lee ${ }^{1 *}$, Muhammad Udin Harun Al Rasyid ${ }^{1}$ and Huai-Kuei Wu ${ }^{2}$
}

\begin{abstract}
Wireless sensor networks based on the IEEE 802.15.4 standard are able to achieve low-power transmissions in the low-rate and short-distance wireless personal area network (PAN). A cluster tree network consists of several clusters; each cluster has a coordinator, known as cluster coordinator, and several device nodes. In the cluster tree topology of IEEE 802.15.4, a PAN coordinator periodically transmits beacon frames to its coordinator nodes as well as a coordinator node periodically transmit beacon frames to their device nodes. The main challenge in the cluster tree network is the collisions between beacons or even between beacon and data frames, which degrades the network performance. In order to decrease collisions, this article proposes the superframe adjustment and beacon transmission scheme (SABTS) by assigning the accurate values of beacon order and superframe order for the PAN coordinator, cluster coordinators, and device nodes, and deciding the precise time for the beacon transmission of PAN and coordinator nodes. A Markov chain model for the cluster tree network is developed with taking into account packet retransmission, acknowledgement, and defer transmission. Both analytical and simulation results show that SABTS performs better than IEEE 802.15.4 standard in terms of the probability of successful transmission, network goodput, and energy consumption.
\end{abstract}

Keywords: Wireless sensor network, IEEE 802.15.4, Personal area network, Cluster tree topology, Markov chain

\section{Introduction}

Wireless sensor networks based on the IEEE 802.15.4 standard have been designed to specify the physical layer (PHY) and medium access control (MAC) sublayer for low power consumption, short transmission range, and low-rate wireless personal area network (LR-WPAN) [1]. The IEEE 802.15.4 standard has three kinds of topology: star, peer-to-peer, and cluster tree topologies, which can operate on beacon- and non-beacon-enabled modes. The beacon-enabled mode has the most unique features of IEEE 802.15.4, while the beacons are used to synchronize the attached devices, to identify the personal area network (PAN), and to describe the structure of the superframe.

A cluster tree network consists of several clusters; each cluster has a coordinator, known as cluster coordinator,

\footnotetext{
* Correspondence: bhlee@mail.ntust.edu.tw

${ }^{1}$ National Taiwan University of Science and Technology, 43, Keelung Rd. Section 4, Taipei 106, Taiwan

Full list of author information is available at the end of the article
}

and several device nodes. A PAN coordinator serves as root to form the first cluster and initiates the network. The PAN coordinator and coordinator nodes broadcast beacon frames to their neighboring devices to complete the whole cluster networks. In a cluster tree topology, the PAN coordinator periodically transmits beacon frames to its coordinator nodes as well as the coordinator nodes periodically transmit beacon frames to their device nodes. However, if the coordinator nodes send beacon frames at the same time, collisions will happen among these beacon frames. Consequently, the children nodes in the cluster cannot synchronize and communicate with their coordinators. The main challenge in the cluster tree network of IEEE 802.15.4 beacon-enabled mode is the collisions between beacons or even between beacon and data frames, which degrades the network performance [2-4]. In other words, it is a crucial challenge on the cluster scheduling and collision avoidance (CA) in a cluster tree network. 
To solve the aforementioned problem, a time division beacon scheduling (TDBS) and superframe duration (SD) scheduling mechanisms are proposed [2]. The idea of TDBS is to manage beacon frame transmission from coordinator nodes in a non-overlapping way while the idea of SD scheduling is to decide the duty cycle of router nodes. A multi-dimensional scheduling (MDS) is proposed to avoid beacon collision in LR-WPAN, which uses the clean channel searching scan to change the time offset to transmit a new beacon frame during the inactive period [3]. MDS can minimize the possibility of beacon collisions, but the power consumption of the PAN coordinator is more than that of IEEE 802.15.4 standard due to the process of channel searching scan during inactive period. Therefore, in order to improve the network performance by decreasing beacon collisions as well as the collisions between beacon and data packets, this article proposes the superframe adjustment and beacon transmission scheme (SABTS) which is based on the IEEE 802.15.4 slotted carrier sense multiple access with CA (CSMA/CA), to assign the accurate values of beacon order (BO) and superframe order (SO) for the PAN coordinator, cluster coordinators and device nodes, and to decide the precise time for beacon transmission of PAN and coordinator nodes.

A number of mathematical analysis models have been proposed to analyze the performance of IEEE 802.15.4 based on the Markov chain model without considering packet retransmissions [5-11]. Several modified Markov chain models including packet retransmissions have been investigated but not consider the defer transmission [12-15], while the authors of [16-18] improve the Markov chain models by considering the defer transmission. An analytical model based on Markov chain for multi-hop cluster network has been studied without taking into account the acknowledgement (ACK) to confirm the successes of data packet transmission, the defer transmission, and packet retransmission [19]. Lastly, we propose an analytical model based on Markov chain for SABTS cluster tree network with taking into account packet retransmission, ACK, and defer transmission by modifying the Markov chain model from [17]. The major contribution of this article is to model the channel access for a cluster tree network to obtain network goodput and energy consumption based on the proposed Markov chain.

\section{Overview of IEEE $\mathbf{8 0 2 . 1 5 . 4}$}

In the beacon-enabled mode of IEEE 802.15.4, each node employs two system parameters: $\mathrm{BO}$ and $\mathrm{SO}$, which define beacon interval (BI) and $\mathrm{SD}$, respectively, i.e., $\mathrm{BI}=$ aBaseSuperframeDuration $\times 2^{\mathrm{BO}}$ and $\mathrm{SD}=\mathrm{aBaseSuper}-$ frameDuration $\times 2^{\mathrm{SO}}$, for $0 \leq \mathrm{SO} \leq \mathrm{BO} \leq 14$. aBaseSuperframeDuration denotes the minimum number of symbols in an active period, which is fixed to 960 symbols. The active period of each superframe consists of three parts: beacon, contention access period (CAP) and contention free period (CFP), while the active period is further equally divided into 16 time slots called aNumSuperframeSlots. The length of one slot is equal to aBaseSlotDuration $\times 2^{\mathrm{SO}}$ symbols, where aBaseSlotDuration is the minimum number of symbols in a slot and equal to 60 symbols. Figure 1 shows an example of the superframe structure. In IEEE 802.15.4 standard, BO and SO shall be equal for all superframes on a PAN. All devices shall interact with the PAN only during the active portion of a superframe.

In CAP, each node performs the CSMA/CA algorithm before transmitting data frame or MAC command frame. Each device maintains three parameters: the number of backoff (NB), contention window $(\mathrm{CW})$, and backoff exponent (BE). NB denotes the required $\mathrm{NB}$ while attempting to transmit data; $C W$ denotes the number of backoff periods that need to be clear before committing transmission; and BE denotes how many backoff periods a device need to wait before trying to access the channel. The initial value of $\mathrm{NB}, \mathrm{CW}$, and $\mathrm{BE}$ are equal to 0,2 , and macMinBE, respectively, where macMinBE is equal to 3 .

In the located boundary of the next backoff period, a device takes delay for random backoff between 0 and $2^{\mathrm{BE}}-1$ unit backoff period (UBP), where UBP is equal to 20 symbols (or 80 bits). A device performs clear channel assessment (CCA) to make sure whether the channel is idle or busy, when the number of random backoff periods is decreased to 0 . The value of CW will be

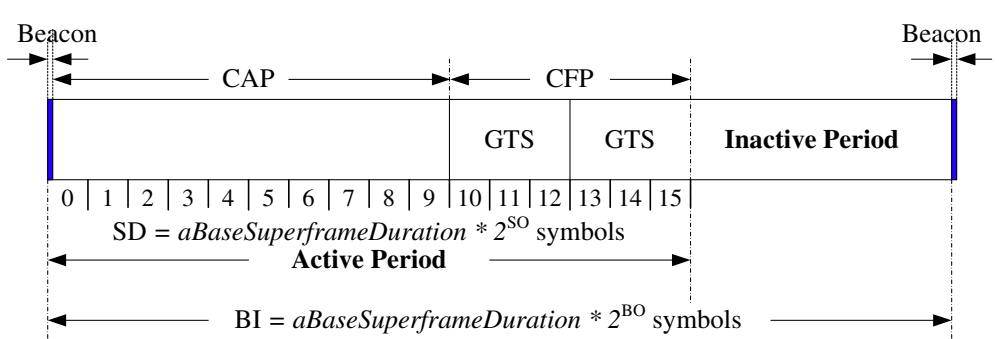

Figure 1 An example of the superframe structure. 
decreased by one if the channel is idle; and the second CCA will be performed if the value of $\mathrm{CW}$ is not equal to 0 . If the value of $C W$ is equal to 0 , it means that the channel is idle after twice CCA; then a device is committed the data transmission. However, if the CCA is busy, the value of $\mathrm{CW}$ will reset to 2 ; the value of $\mathrm{NB}$ is increased by 1 ; and the value of $\mathrm{BE}$ is increased by 1 up to the maximum $\mathrm{BE}$ (macMaxBE), where the value macMaxBE is equal to 5 . The device will repeatedly take random delay if the value of $\mathrm{NB}$ is less than the value of macMaxCSMABackoff, where the value of macMaxCSMABackoff is equal to 4; and the transmission attempt is decided to be failure if the value of $\mathrm{NB}$ is greater than the value of macMaxCSMABackoff.

\section{Description of SABTS}

SABTS aims to assign the accurate values of $\mathrm{BO}$ and $\mathrm{SO}$ for the PAN coordinator, cluster coordinators and device nodes, and to decide the precise time for the beacon transmission of PAN and coordinator nodes. In order to guarantee the data transmission from its coordinator nodes, the BI of the PAN coordinator should be the round function to the interarrival time (INTV) of data packets. Let us denote $B O_{\text {PAN }}$ be the $\mathrm{BO}$ for the PAN coordinator, which can be obtained by Equation 1, where $N_{\text {coord }}$ denotes the number of coordinator nodes; $R s, B s$, and $N s$ denote symbol rate, aBaseSlotDuration, and aNumSuperframeSlots, respectively, e.g., Rs, $B s$, and $N s$ are equal to 62,500 symbols/s, 60 symbols, and 16 slots, respectively. To reduce the beacon collisions between the parent and children coordinators, the different $\mathrm{BO}$ between coordinators at different depth can be obtained by Equation 2, where $B O_{\text {coord }}$ is the $\mathrm{BO}$ for coordinator. The PAN coordinator might often be powered; therefore, the $\mathrm{SO}$ for the PAN coordinator $\left(\mathrm{SO}_{\mathrm{PAN}}\right)$ can be set to its $B O_{\mathrm{PAN}}$ as shown in Equation 3. For the SD, if we assume there is no CFP, the SD of coordinator $\left(S D_{\text {coord }}\right)$ only consists of CAP and beacon as defined in Equation 4, where Estimated $C A P_{\text {coord }}$ and $L_{\text {beacon }}$ denote the estimated CAP for coordinator and the length of beacon, respectively, e.g., $L_{\text {beacon }}$ is equal to 190 symbols.

$$
\begin{aligned}
& B O_{\mathrm{PAN}}=\left\lfloor\left(\log _{2}\left(\frac{N_{\text {coord }} \times I N T V \times R s}{B s \times N s}\right)\right)\right\rfloor \\
& B O_{\text {coord }}=B O_{\text {PAN }}-1 \\
& S O_{\text {PAN }}=B O_{\text {PAN }} \\
& S D_{\text {coord }}=\text { EstimatedCAP }_{\text {coord }}+L_{\text {Beacon }}
\end{aligned}
$$

EstimatedCAP $P_{\text {coord }}$ is equal to the $\mathrm{BI}$ of coordinator $\left(B I_{\text {coord }}\right)$ divided by the number of coordinator nodes $\left(N_{\text {coord }}\right)$, as shown in Equation 5 . By using Equations 4 and 5, $S D_{\text {coord }}$ can be obtained by Equation 6 . Therefore, the value of $\mathrm{SO}$ for coordinator $\left(\mathrm{SO}_{\text {coord }}\right)$ can be obtained by Equation 7. BO and $\mathrm{SO}$ for device nodes $\left(B O_{\mathrm{dev}}\right.$ and $\left.S \mathrm{SO}_{\mathrm{dev}}\right)$ are decided by its coordinator node, which are equal to $\mathrm{BO}$ and $\mathrm{SO}$ of its coordinator node as shown in Equation 8.

$$
\begin{array}{r}
\text { EstimatedCAP } P_{\text {coord }}=\frac{B I_{\text {coord }}}{N_{\text {coord }}} \\
=\frac{B s \times N s \times 2^{B O_{\text {coord }}}}{N_{\text {coord }}} \\
S D_{\text {coord }}=B s \times N s \times 2^{S O \text { coord }} \\
=\frac{B s \times N s \times 2^{B O_{\text {coord }}}+190}{N_{\text {coord }}}+10 \\
S O_{\text {coord }}=\left\lfloor\log _{2}\left(\frac{2^{B O_{\text {coord }}}}{N_{\text {coord }}}+0.2\right)\right\rfloor \\
B O_{d e v}=B O_{\text {coord }} ; S O_{d e v}=S O_{\text {coord }}
\end{array}
$$

Based on the aforementioned description, SABTS can be resumed by flowchart as shown in Figure 2. In this article, we consider the cluster tree topology with one PAN, three coordinator nodes, and nine device nodes as shown in Figure 3. Figure 4 shows an example of the superframe adjustment and precise time for beacon transmission for the cluster tree topology of Figure 3,

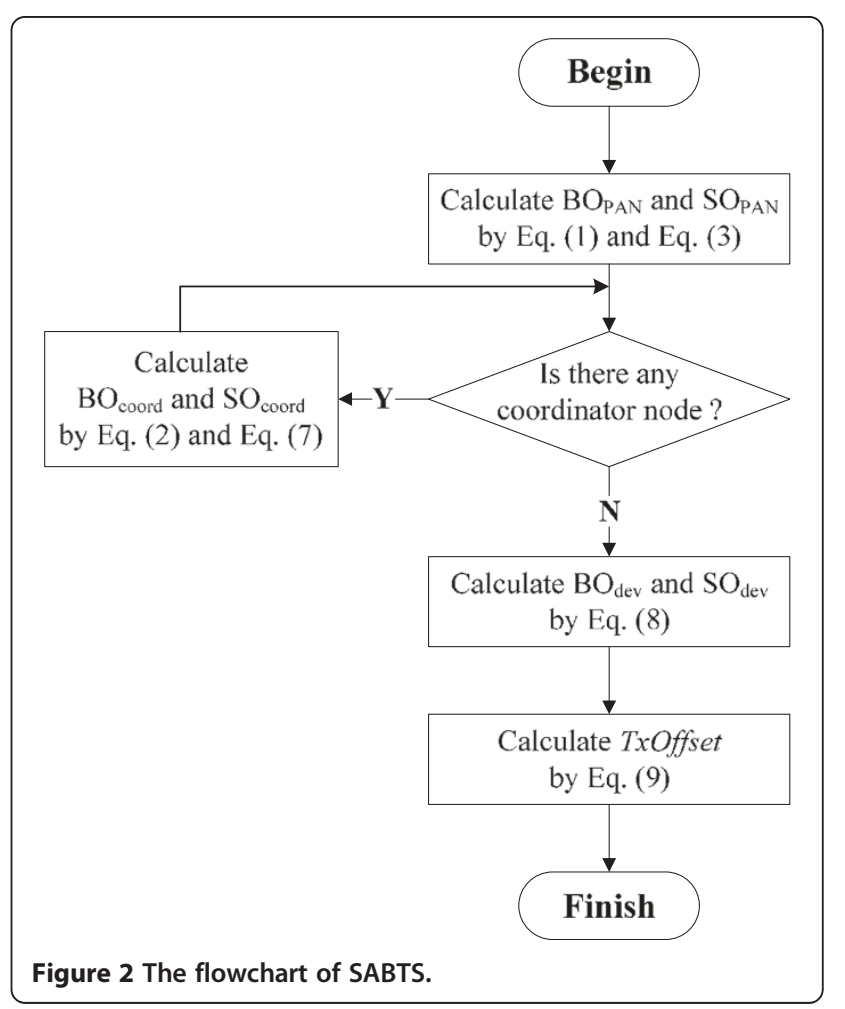




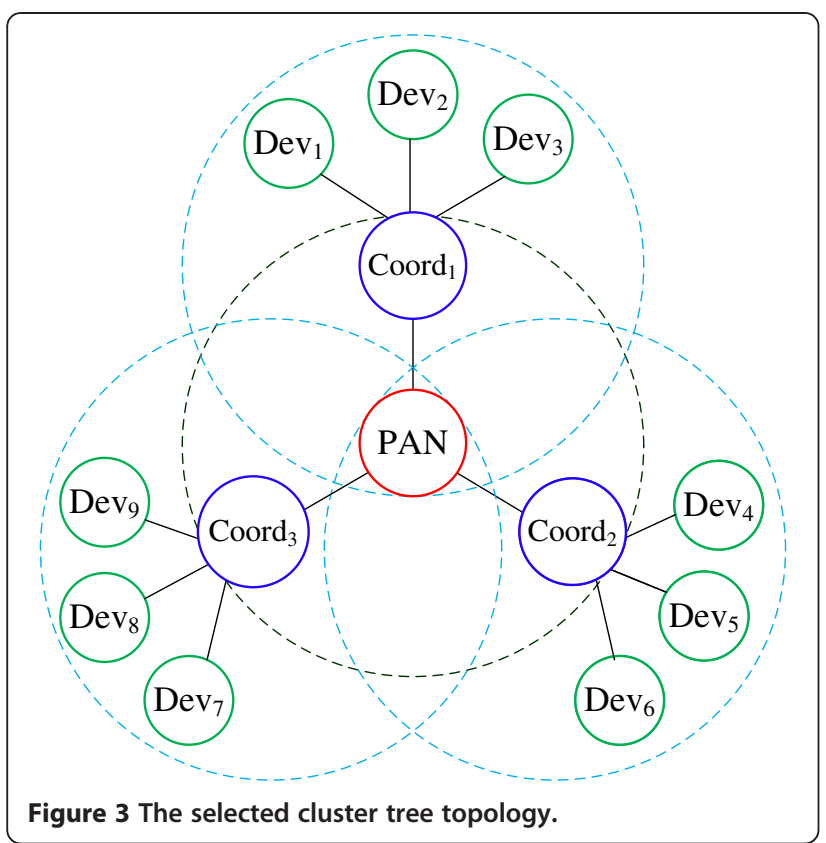

while INTV of each device node is equal to 0.1. As shown in Figure 4, we get the different starting time of beacon transmission among coordinator nodes to avoid beacon collisions. Furthermore, the coordinator nodes and device nodes can save energy consumption during inactive periods. By using SABTS, we get the values of $\mathrm{BO}$ and $\mathrm{SO}$ for PAN to be equal to 4; the value of $\mathrm{BO}$ for each coordinator node and device node is equal to 3 ; and the value of SO for each coordinator node and device node is equal to 1 .
In order to reduce the collisions of the beacon transmissions among coordinator nodes, SABTS adjusts the beacon starting times of PAN and coordinator nodes. Let us denote $T_{x} O f f s e t_{\text {PAN }}$ and $T_{x} O_{f f s e t}$ to be the beacon starting times of PAN and the $i$ th coordinator node, respectively. TxOffset ${ }_{\mathrm{PAN}}$ starts at the beginning of superframe, then $T_{x} O f f s e t_{i}$ is adjusted by Equation 9, where $S D_{\text {coord }_{i-1}}$ denotes the SD of the $(i-1)$ th coordinator node.

$$
T_{x} \text { Offset }_{i}=\left\{\begin{array}{l}
\text { TxOffset } \\
\text { PAN } \\
T_{x O f f s e t}+\frac{L_{\text {Beacon }}}{R s}, i=1 \\
2 \leq i \leq N_{\text {coord }}
\end{array}\right.
$$

\section{Analysis of SABTS}

In this section, the Markov chain model for SABTS in the case of the acknowledged uplink data transmission is analyzed to obtain the stationary probabilities, whose state transition diagram is shown as Figure 5 . Let $b_{i, j, k}$ be the stationary probability at the stochastic state $(s(t)=i$, $c(t)=j$, and $r(t)=k)$, where $s(t), c(t)$, and $r(t)$ represent backoff stage, backoff counter, and number of retransmissions, respectively, shown as Equation 10, where $b_{i,-1, k}$ $b_{i,-2, k}$ and $b_{i,-3, k}$ are the stationary probabilities for the first $\mathrm{CCA}_{1}$, the second $\mathrm{CCA}_{2}$, and packet transmission, respectively, at the $i$ th backoff stage and the $k$ th retransmission. Let $b_{S i, k}$ and $b_{C i, k}$ be the stationary probabilities of the successful transmission and collision at the states of $S_{i, k}$ and $C_{i, k}$ shown as Equations (11) and (12), respectively, where $m$ and $R$ are the maximum NB

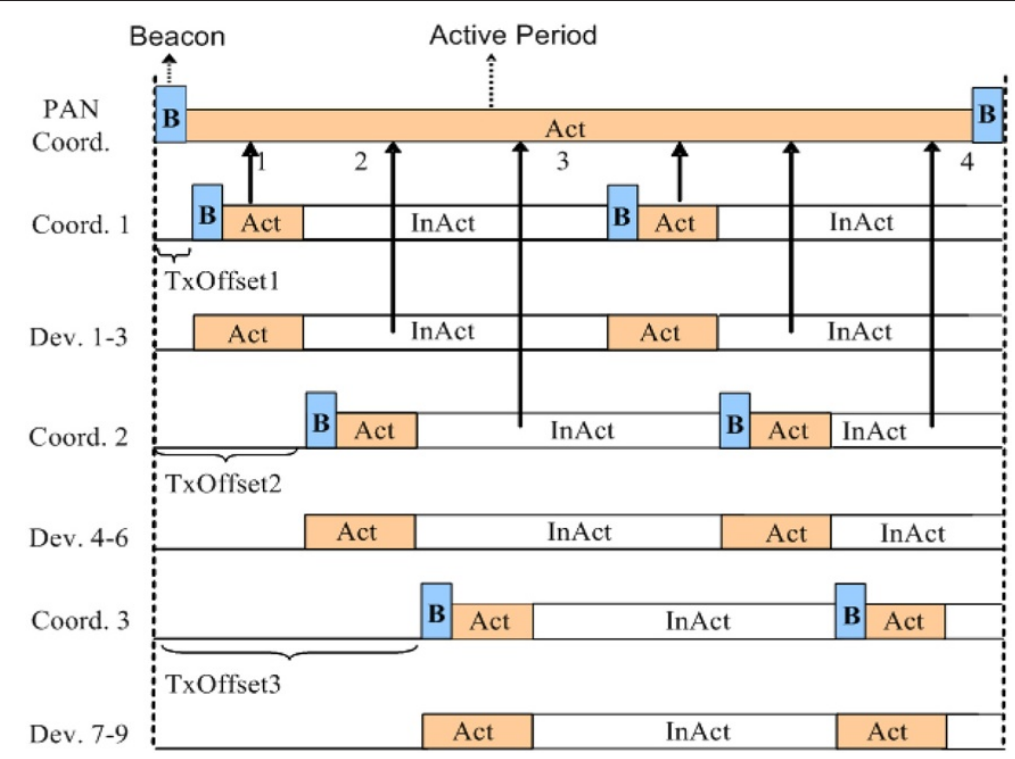

Figure 4 An example of SABTS for three coordinator nodes. 


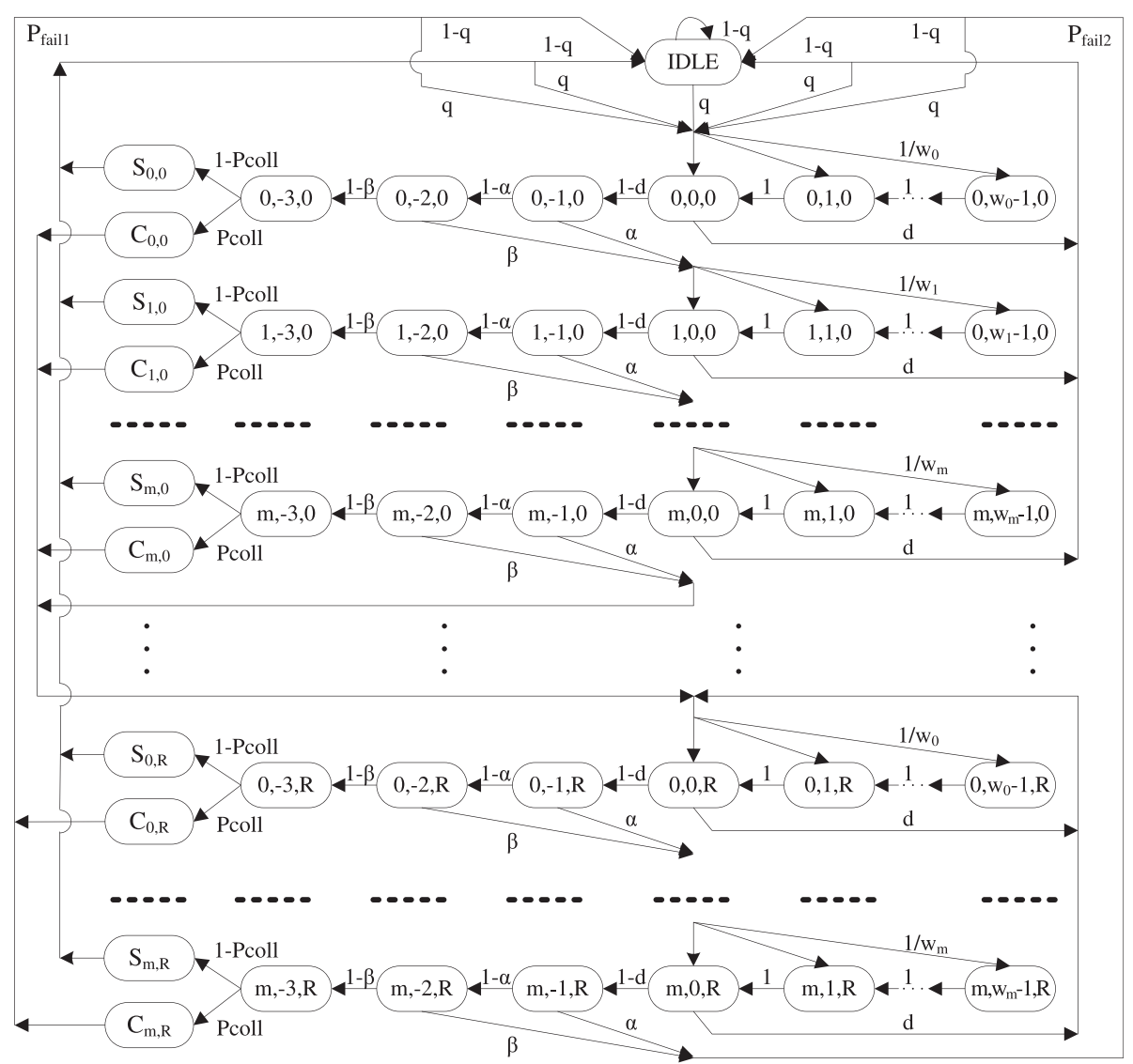

Figure 5 The Markov chain model for CSMA/CA.

stage and retransmissions, i.e., they are equal to 4 and 3 , respectively.

$$
\begin{aligned}
b_{i, j, k}= & \lim _{t \rightarrow \infty} P\{s(t)=i, c(t)=j, r(t)=k\}, \\
& \text { for } i \in(0, \mathrm{~m}), j \in\left(-3, \mathrm{w}_{i}-1\right), k \in(0, R) \\
b_{S_{i, k}}= & \lim _{t \rightarrow \infty} P\left\{S_{s(t)}=S_{i}, r(t)=k\right\}, i \in(0, \mathrm{~m}), k \in(0, R) \\
b_{C_{i, k}}= & \lim _{t \rightarrow \infty} P\left\{C_{s(t)}=C_{i}, r(t)=k\right\}, i \in(0, m), k \in(0, R)
\end{aligned}
$$

The parameters used in the Markov chain model are explained as follows. An IDLE state means that a device node has no packet to transmit. Let $w_{i}=2^{B E_{i}}$ be the backoff window at the $i$ th backoff stage of a device, where $\mathrm{BE}_{i}=3,4,5,5$, and 5 for $0 \leq i \leq m$. Let us denote $q$ to be the probability that packet arrives during the active period, which can be obtained by Equation 13, where $L_{\text {data }}, R_{b}$, and $S D T$ are packet length (in bits), data rate (i.e., $250 \mathrm{kbps}$ ), and the SD time (in seconds), respectively; where $S D T$ is equals to $\frac{S D}{R s}$, and $\frac{1}{I N T V}$ is the number of packets in $S D T$.

$$
q=\frac{L_{\text {data }}}{I N T V \times R_{b} \times S D T}
$$

The MAC sublayer should transmit packet if the remaining CSMA/CA steps, i.e., two CCA analyses, frame transmission, and any $\mathrm{ACK}$, can be completed before the end of CAP. Conversely, if the current CAP has not enough slots to transmit data packets, it should defer transmission until the beginning of the CAP in the next superframe. Let us denote $d$ to be the probability of defer transmission, which can be obtained by Equation 14 , where $T_{t x c c a}, T_{t x}, T_{\text {ack }}$, and $T_{t x a c k}$ are the CCA transmission time, packet transmission time, time to wait for $\mathrm{ACK}$, and time to transmit ACK from receiver to transmitter node, respectively.

$$
d=\frac{2 T_{t x c c a}+T_{t x}+T_{\text {ack }}+T_{\text {txack }}}{S D T}
$$

Let $\alpha$ and $\beta$ be the probabilities that $\mathrm{CCA}_{1}$ and $\mathrm{CCA}_{2}$ are busy, respectively. $\mathrm{CCA}_{1}$ busy means that the tagged 
node at one of the $\mathrm{CCA}_{1}$ states while at least one of the other nodes at packet transmission state, while $\mathrm{CCA}_{2}$ busy means that the tagged node at one of the $\mathrm{CCA}_{2}$ states while at least one of the other nodes at packet transmission state. Let us denote $P_{\text {coll }}$ to be the probability of the collision of packet transmission, i.e., the tagged node at packet transmission state while at least one of other nodes in the packet transmission state at the same time. Let us also denote $P_{\text {fail1 }}$ and $P_{\text {fail } 2}$ to be the probabilities of fail transmission due to the maximum number of retransmissions after collisions and due to no channel to use after reaching the maximum backoff stage at the maximum retransmission stage, respectively.

To analyze the Markov chain model, several state transition probabilities are evaluated as shown from Equations 15 to 23 . Equation 15 states the probability that the backoff counter is decreased after each slot. Equation 16 gives the probability of finding busy channel either in $\mathrm{CCA}_{1}$ and $\mathrm{CCA}_{2}$. Equation 17 states the probability of picking a backoff state in the next retransmission stage after the collision of packet transmission when having enough time to send packet in the remaining active period and channel idle in both $\mathrm{CCA}_{1}$ and $\mathrm{CCA}_{2}$. Equation 18 states the probability of entering the IDLE state after the collision of packet transmission while reach the maximum retransmission stage after finding the remaining active period to be enough to send packet and channel idle in both $\mathrm{CCA}_{1}$ and $\mathrm{CCA}_{2}$. Equation 19 gives probability that the remaining $\mathrm{CAP}$ is not enough to send packet and need to defer and pick backoff state in the next superframe. Equation 20 states the probability of successful packet transmission and picking new random backoff at the first backoff stage. Equation 21 states the probability of entering the IDLE state if the node has no data packet to transmit after successful packet transmission. Equation 22 states the probability of entering the IDLE state due to channel access failure. Equation 23 states the probability of going to the first backoff stage from the IDLE state if the node has data packet to transmit.

$$
\begin{aligned}
P(i, j, k \mid i, j+1, k)= & 1, \text { for } i \in(0, m), j \in\left(0, w_{i}-2\right), \\
& k \in(0, R)
\end{aligned}
$$

$$
\begin{aligned}
P(i, j, k \mid i-1,0, k)= & \frac{w_{i}-j}{w_{i}}[(1-d)(\alpha)+(1-d)(1-\alpha) \beta] \\
= & \frac{w_{i}-j}{w_{i}}(1-d)[\alpha+(1-\alpha) \beta], \\
& i \in(1, m), j \in\left(0, w_{i}-1\right), k \in(0, R)
\end{aligned}
$$

$$
\begin{gathered}
P(0,0, k \mid i, 0, k-1)=(1-d)(1-\alpha)(1-\beta)\left(P_{\text {coll }}\right), \\
k \in(1, R) \\
P(I D L E \mid i, 0, R)=(1-d)(1-\alpha)(1-\beta)\left(P_{\text {coll }}\right)(1-q)
\end{gathered}
$$

$$
\begin{aligned}
& P(0, j, k \mid i, 0, k)=\frac{w_{0}-j}{w_{0}}(d), k \in(0, R) \\
& P(0, j, 0 \mid i, 0, k) \\
& =\frac{w_{0}-j}{w_{0}}(1-d)(1-\alpha)(1-\beta)\left(1-P_{\text {coll }}\right)(q), \\
& \quad i \in(0, m), j \in\left(0, w_{i}-1\right), k \in(0, R)
\end{aligned}
$$

$$
\begin{aligned}
& P(I D L E \mid i, 0, k) \\
& =(1-d)(1-\alpha)(1-\beta)\left(1-P_{\text {coll }}\right)(1-q), \\
& \quad i \in(0, m), k \in(0, R)
\end{aligned}
$$

$$
P(I D L E \mid m, 0, R)=(1-d)[\alpha+(1-\alpha) \beta](1-q)
$$

$$
P(0, j, 0 \mid I D L E)=\frac{w_{0}-j}{w_{0}}(q), j \in\left(0, w_{i}-1\right)
$$

By using Equation 16, the stationary probability $b_{i j, j}$ can be obtained by Equation 24. From Equation 17, $b_{0,0, k}$ can be obtained by Equation 25, where $Y$ and $X$ are the probabilities of entering the next backoff stage and the collision of packet transmission in a certain backoff stage, respectively. Similarly, $b_{i, 0, k}$ can be obtained by Equation 26. Finally, the steady-state probabilities to perform random backoff, $\mathrm{CCA}_{1}, \mathrm{CCA}_{2}$, packet transmission, successful of packet transmission, collision of packet transmission, and idle can be obtained from Equations 27 to 33, respectively. Since the sum of probabilities in the Markov chain must be equal to one, we have Equation 34. By using Equations 27 to 34, we can get the value of $b_{0,0,0}$ easily by using excel spreadsheet.

$$
\begin{aligned}
b_{i, j, k} & =\frac{w_{i}-j}{w_{i}} b_{i-1,0, k}(1-d)[\alpha+(1-\alpha) \beta] \\
& =\frac{w_{i}-j}{w_{i}} b_{i, 0, k}
\end{aligned}
$$




$$
\begin{aligned}
b_{0,0, k}= & (1-d)(1-\alpha)(1-\beta) P_{\text {coll }} \sum_{i=0}^{m} b_{i, 0, k-1} \\
& +(1-d)[\alpha+(1-\alpha) \beta] b_{m, 0, k-1} \\
= & (1-d)(1-\alpha)(1-\beta) P_{\text {coll }} \sum_{i=0}^{m} b_{i, 0, k-1}+\mathrm{Y} b_{m, 0, k-1} \\
= & b_{0,0,0}\left[\left((1-d)(1-\alpha)(1-\beta) P_{\text {coll }} \sum_{i=0}^{m} \mathrm{Y}^{i}\right)^{k}\right. \\
& \left.+\left(\mathrm{Y}^{m+1}\right)^{k}\right] \\
= & b_{0,0,0}\left[\left((1-d)(1-\alpha)(1-\beta) P_{\text {coll }}\left(\frac{1-\mathrm{Z}}{1-\mathrm{Y}}\right)\right)^{k}+\mathrm{Z}^{k}\right] \\
= & b_{0,0,0}\left[\left((1-d)(1-\alpha)(1-\beta) P_{\text {coll }} \mathrm{U}\right)^{k}+\mathrm{Z}^{k}\right] \\
= & b_{0,0,0}\left(\mathrm{X}^{k}+\mathrm{Z}^{k}\right)
\end{aligned}
$$

where $Y=(1-d)[\alpha+(1-\alpha) \beta] ; Z=Y^{m+1} ; U=\left(\frac{1-Z}{1-Y}\right)$; and $X=(1-d)(1-\alpha)(1-\beta) P_{\text {coll }} U$ for simplicity.

$$
\begin{aligned}
b_{i, 0, k} & =b_{i-1,0, k}(1-d)[\alpha+(1-\alpha) \beta] \\
& =((1-d)[\alpha+(1-\alpha) \beta])^{i} b_{0,0, k} \\
& =Y^{i} b_{0,0, k} \\
& =Y^{i} b_{0,0,0}\left(X^{k}+Z^{k}\right) \text { for } i \in(0, m), k \in(0, R)
\end{aligned}
$$

$$
\begin{aligned}
P[\text { random backoff }]= & \sum_{i=0}^{m} \sum_{j=0}^{w_{i}-1} \sum_{k=0}^{R} b_{i, j, k} \\
= & \sum_{i=0}^{m} \sum_{k=0}^{R} \frac{w_{i}}{2} \mathrm{Y}^{i} b_{0,0, k} \\
= & \frac{w_{0}}{2} b_{0,0,0}\left(\frac{1-(\mathrm{X}+\mathrm{Z})^{R}}{1-(\mathrm{X}+\mathrm{Z})}-2 \mathrm{XZ}\right) \\
& \times\left[\frac{1-(2 \mathrm{Y})^{R}}{1-(2 \mathrm{Y})}+4 \mathrm{Y}^{3}+4 \mathrm{Y}^{4}\right] \\
= & \frac{w_{0}}{2} b_{0,0,0} \mathrm{~V} \mathrm{Q}
\end{aligned}
$$

where $V=\frac{1-(X+Z)^{R}}{1-(X+Z)}-2 X Z$ and $\mathrm{Q}=\frac{1-(2 Y)^{R}}{1-(2 Y)}+4 Y^{3}+4 Y^{4}$ for simplicity.

$$
\begin{aligned}
p\left[C C A_{1}\right] & =\sum_{i=0}^{m} \sum_{k=0}^{R} b_{i,-1, k} \\
& =(1-d) \sum_{i=0}^{m} \sum_{k=0}^{R} \mathrm{Y}^{i} b_{0,0, k} \\
& =b_{0,0,0}\left(\frac{1-(\mathrm{X}+\mathrm{Z})^{R}}{1-(\mathrm{X}+\mathrm{Z})}-2 \mathrm{XZ}\right)\left(\frac{1-\mathrm{Z}}{1-\mathrm{Y}}\right)(1-d) \\
& =b_{0,0,0} \mathrm{~V} \mathrm{U}(1-d)
\end{aligned}
$$

$$
\begin{aligned}
P\left[C C A_{2}\right]= & \sum_{i=0}^{m} \sum_{k=0}^{R} b_{i,-2, k} \\
= & (1-d)(1-\alpha) \sum_{i=0}^{m} \sum_{k=0}^{R} \mathrm{Y}^{i} b_{0,0, k} \\
= & b_{0,0,0}\left(\frac{1-(\mathrm{X}+\mathrm{Z})^{R}}{1-(\mathrm{X}+\mathrm{Z})}-2 \mathrm{XZ}\right)\left(\frac{1-\mathrm{Z}}{1-\mathrm{Y}}\right) \\
& \times(1-d)(1-\alpha) \\
= & b_{0,0,0} \mathrm{~V} \mathrm{U}(1-d)(1-\alpha)
\end{aligned}
$$

$P[$ packet transmission $]$

$$
\begin{aligned}
= & \sum_{i=0}^{m} \sum_{k=0}^{R} b_{i,-3, k} \\
= & (1-d)(1-\alpha)(1-\beta) \sum_{i=0}^{m} \sum_{k=0}^{R} \mathrm{Y}^{i} b_{0,0, k} \\
= & b_{0,0,0}\left(\frac{1-(\mathrm{X}+\mathrm{Z})^{R}}{1-(\mathrm{X}+\mathrm{Z})}-2 \mathrm{XZ}\right)\left(\frac{1-\mathrm{Z}}{1-\mathrm{Y}}\right)(1-d) \\
& \times(1-\alpha)(1-\beta)=b_{0,0,0} \mathrm{~V} \mathrm{U}(1-d)(1-\alpha) \\
& \times(1-\beta)
\end{aligned}
$$

$P[$ successful of packet transmission $]$

$$
\begin{aligned}
= & \sum_{i=0}^{m} \sum_{k=0}^{R} b_{S_{i, k}} \\
= & (1-d)(1-\alpha)(1-\beta)\left(1-P_{\text {coll }}\right) \sum_{i=0}^{m} \sum_{k=0}^{R} \mathrm{Y}^{i} b_{0,0, k} \\
= & b_{0,0,0}\left(\frac{1-(\mathrm{X}+\mathrm{Z})^{R}}{1-(\mathrm{X}+\mathrm{Z})}-2 \mathrm{XZ}\right)\left(\frac{1-\mathrm{Z}}{1-\mathrm{Y}}\right)(1-d) \\
& \times(1-\alpha)(1-\beta)\left(1-P_{\text {coll }}\right) \\
= & b_{0,0,0} \mathrm{~V} \mathrm{U}(1-d)(1-\alpha)(1-\beta)\left(1-P_{\text {coll }}\right)
\end{aligned}
$$

\section{$P[$ collision of packet transmission $]$}

$$
\begin{aligned}
& =\sum_{i=0}^{m} \sum_{k=0}^{R} b_{C_{i, k}} \\
& =(1-d)(1-\alpha)(1-\beta) P_{\text {coll }} \sum_{i=0}^{m} \sum_{k=0}^{R} \mathrm{Y}^{i} b_{0,0, k} \\
& =b_{0,0,0} \mathrm{X}\left(\frac{1-(\mathrm{X}+\mathrm{Z})^{R}}{1-(\mathrm{X}+\mathrm{Z})}-2 \mathrm{XZ}\right) \\
& =b_{0,0,0} \mathrm{XV}
\end{aligned}
$$




$$
\begin{aligned}
& P[I D L E]=(1-q) \sum_{i=0}^{m} \sum_{k=0}^{R} b_{S_{i, k}}+(1-q) \sum_{i=0}^{m} b_{C_{i, R}}+(1-q) d \sum_{i=0}^{m} b_{i, 0,0}+(1-q) \mathrm{Y} b_{m, 0, R}+(1-q) P[I D L E] \\
&=\frac{(1-q)}{q}\left[\sum_{i=0}^{m} \sum_{k=0}^{R} b_{S_{i, k}}+\sum_{i=0}^{m} b_{C_{i, R}}+d \sum_{i=0}^{m} b_{i, 0,0}+\mathrm{Y} b_{m, 0, R}\right] \\
&=\frac{(1-q)}{q} b_{0,0,0}\left[\begin{array}{l}
\left.\left(\frac{1-(\mathrm{X}+\mathrm{Z})^{R}}{1-(\mathrm{X}+\mathrm{Z})}-2 \mathrm{XZ}\right)\left(\frac{1-\mathrm{Z}}{1-\mathrm{Y}}\right)(1-d)(1-\alpha)(1-\beta)\left(1-P_{\text {coll }}\right)\right] \\
+\left(\mathrm{X}^{2}+\mathrm{Z}^{2}\right) \mathrm{X}+d\left(\frac{1-\mathrm{Z}}{1-\mathrm{Y}}\right)+\mathrm{Z}\left(\mathrm{X}^{2}+\mathrm{Z}^{2}\right)
\end{array}\right] \\
&= \frac{(1-q)}{q} b_{0,0,0}\left[V U(1-d)(1-\alpha)(1-\beta)\left(1-P_{\text {coll }}\right)+\left(X^{2}+Z^{2}\right)(X+Z)+d U\right] \\
& \sum_{i=0}^{m} \sum_{j=0}^{w_{i}-1} \sum_{k=0}^{R} b_{i, j, k}+\sum_{i=0}^{m} \sum_{k=0}^{R} b_{i,-1, k}+\sum_{i=0}^{m} \sum_{k=0}^{R} b_{i,-2, k}+\sum_{i=0}^{m} \sum_{k=0}^{R} b_{i,-3, k}+\sum_{i=0}^{m} \sum_{k=0}^{R} b S_{i, k} \\
&+\sum_{i=0}^{m} \sum_{k=0}^{R} b C_{i, k}+P[I D L E]=1
\end{aligned}
$$

Let $\phi_{1}$ and $\phi_{2}$ be the conditional probabilities that a tagged node will be at one of the CCA states after backoff and at one of the $\mathrm{CCA}_{2}$ states after sensing channel idle in the $\mathrm{CCA}_{1}$, which can be obtained by Equations 35 and 36, respectively. Let us denote $\tau$ to be the probability that a tagged node can transmit a packet, i.e., the tagged node is in one of the $C C A_{1}$ states and senses the $C C A_{2}$ is idle, while the other nodes do not in the $C C A_{1}$ state, which can be expressed by Equation 37, where $N_{\mathrm{ch}}$ is the number of children nodes of certain coordinator nodes. Therefore, the previous mentioned probabilities of $\alpha, \beta, P_{\text {coll }}, P_{\text {fail } 1}$, and $P_{\text {fail2 }}$ can be expressed by Equations 38 to 42, respectively.

$$
\begin{aligned}
\phi_{1} & =\frac{\sum_{i=0}^{m} \sum_{k=0}^{R} b_{i,-1, k}}{\sum_{i=0}^{m} \sum_{k=0}^{R} b_{i,-1, k}+\sum_{i=0}^{m} \sum_{j=0}^{w_{i}-1} \sum_{k=0}^{R} b_{i, j, k}} \\
& =\frac{\left(\frac{1-(X+Z)^{R+1}}{1-(X+Z)}-2 X Z\right)\left(\frac{1-Z}{1-Y}\right)(1-d)}{\left(\frac{1-(X+Z)^{R+1}}{1-(X+Z)}-2 X Z\right)\left[\left(\frac{1-Z}{1-Y}\right)(1-d)+\frac{w_{0}}{2}\left(\frac{1-(2 Y)^{R+1}}{1-(2 Y)}+4 Y^{3}+4 Y^{4}\right)\right]} \\
& =\frac{V^{\prime} U(1-d)}{V^{\prime}\left[U(1-d)+\frac{w_{0}}{2} Q^{\prime}\right]}
\end{aligned}
$$

where $V^{\prime}=\frac{1-(X+Z)^{R+1}}{1-(X+Z)}-2 X Z$ and $Q^{\prime}=\frac{1-(2 Y)^{R+1}}{1-(2 Y)}+4 Y^{3}+4 Y^{4}$ for simplicity.

$$
\begin{aligned}
\phi_{2} & =\frac{\sum_{i=0}^{m} \sum_{k=0}^{R} b_{i,-2, k}}{\sum_{i=0}^{m} \sum_{k=0}^{R} b_{i,-1, k}+\sum_{i=0}^{m} \sum_{k=0}^{R} b_{i,-2, k}+\sum_{i=0}^{m} \sum_{j=0}^{w_{i}-1} \sum_{k=0}^{R} b_{i, j, k}} \\
& =\frac{\left(\frac{1-(X+Z)^{R+1}}{1-(X+Z)}-2 X Z\right)\left(\frac{1-Z}{1-Y}\right)(1-d)(1-\alpha)}{\left(\frac{1-(X+Z)^{R+1}}{1-(X+Z)}-2 X Z\right)\left[\left(\frac{1-Z}{1-Y}\right)(1-d)(2-\alpha)+\left(\frac{w_{0}}{2}\left(\frac{1-(2 Y)^{R+1}}{1-(2 Y)}+4 Y^{3}+4 Y^{4}\right)\right)\right]} \\
& =\frac{V^{\prime} U(1-d)(1-\alpha)}{V^{\prime}\left[U(1-d)(2-\alpha)+\frac{w_{0}}{2} Q^{\prime}\right]}
\end{aligned}
$$




$$
\begin{aligned}
& \tau=\phi_{1}\left(1-\phi_{1}\right)^{N_{\mathrm{ch}}-1}(1-\alpha)(1-\beta) \\
& \alpha=\phi_{1}\left(1-(1-\tau)^{\left(\left(N_{c h}-1\right)\left(\frac{1}{I N T V}\right)\right)}\right) \\
& \beta=\phi_{2}\left(1-(1-\tau)^{\left(\left(N_{c h}-1\right)\left(\frac{1}{I N T V}\right)\right)}\right) \\
& P_{\text {coll }}=N_{c h} \tau\left(1-(1-\tau)^{\left(\left(N_{c h}-1\right)\left(\frac{1}{I N T V}\right)\right)}\right) \\
& P_{\text {faill }}=\sum_{i=0}^{m} b_{C_{i, R}}=b_{0,0,0}\left(X^{R}+Z^{R}\right) X \\
& \begin{array}{l}
P_{\text {fail }}= \\
\quad=b_{m, 0, R}(1-d)(\alpha+(1-\alpha) \beta) \\
=b_{0,0,0}\left(\mathrm{Y}^{m}\right)\left(\mathrm{X}^{R}+\mathrm{Z}^{R}\right)(1-d)(\alpha+(1-\alpha) \beta)
\end{array}
\end{aligned}
$$

Let us denote $P_{d r}$ to be the probability of a packet being dropped due to collision retransmission, which can be expressed by Equation 43. Because the probability of collision increases as the value of INTV decreases, we define $P_{I N T V}$ be the fraction time of the total number of data packets and ACK packets to the CAP time of the superframe as expressed by Equation 44, where interframe space (IFS) is the minimum period between two successive frames transmitted from device [1]. Let us denote $P_{\text {dropcoord }}$ and $P_{\text {succoord }}$ to be the probabilities of packet dropped and successful transmission from device node to its coordinator, respectively, which can be obtained by Equations 45 and 46. Similarly, let us denote $P_{\text {dropPAN }}$ and $P_{\text {sucPAN }}$ be the probabilities of packet dropped and successful transmission from coordinator node to the PAN coordinator, respectively, which can be obtained by Equations 47 and 48 .

$$
\begin{aligned}
& P_{d r}=\sum_{k=1}^{R}\left(P_{\text {coll }}\right)^{k} \\
& P_{I N T V}=\left(\frac{L_{\text {data }}+I F S+A C K}{I N T V \times S D T}\right)^{N_{c h}-1} \\
& P_{\text {dropcoord }}=\left\{\begin{array}{l}
P_{\text {faill }}+P_{\text {fail } 2}+P_{d r}, \text { if } P_{I N T V}<1 \\
1, \text { if } P_{I N T V}=1
\end{array}\right. \\
& P_{\text {succoord }}=1-P_{\text {dropcoord }} \\
& P_{\text {dropPAN }}=\left(P_{\text {dropcoord }}\right)^{2}
\end{aligned}
$$

$$
P_{\text {sucPAN }}=\left(1-P_{\text {dropcoord }}\right)\left(1-P_{\text {dropPAN }}\right)
$$

Based on the aforementioned analyses, the number of successful packets received by PAN ( $\left.N_{\text {recvPAN }}\right)$ and the network goodput $(G)$, can be expressed by Equations 49 and 50, respectively, where $N_{\text {dev }}, N_{\text {beacon }}$, and $T_{\text {sim }}$ are the number of device nodes in network, the number of observed BIs of PAN, and the observed simulation time (in seconds), respectively.

$$
\begin{aligned}
& N_{\text {recvPAN }}=\frac{N_{\text {dev }} P_{\text {sucPAN }} T_{\text {sim }}}{I N T V} \\
& G=\frac{N_{\text {recvPAN }} L_{\text {data }}}{N_{\text {beacon }} B I_{\text {PAN }}}
\end{aligned}
$$

Basically, the total energy consumption of a cluster tree network consists of the energy consumptions by device nodes, coordinator node, and the PAN coordinator, denoted by $E_{\mathrm{dev}}, E_{\mathrm{coord}}$, and $E_{\mathrm{PAN}}$, respectively. Let us denote $P W_{\text {idle, }} P W_{\text {sleep }}, P W_{t x}$, and $P W_{r x}$ to be the power consumptions for idle, sleeping, transmitting a packet, and receiving a packet, respectively. Let us also denote $L_{\text {cca }}, L_{\text {beacon}}$, and $L_{\text {ack }}$ to be the lengths of CCA, beacon, and ACK transmissions, respectively. Moreover, let us denote $D C_{\mathrm{dev}}, D C_{\text {coord }}$, and $D C_{\mathrm{PAN}}$ to be the duty cycles of device nodes, coordinator nodes, and the PAN coordinator, respectively.

The energy consumptions of a cluster tree network are analyzed as follows. The energy consumption of device nodes consist of seven parts as shown in Equation 51, i.e., the seven parts of energy consumptions are in order for backoff, CCA transmission, data packet transmission, idle, receiving beacon from its coordinator, receiving $\mathrm{ACK}$, and sleeping, respectively, where $d_{\text {node }}$ is the distance between device nodes (or coordinator nodes) and its coordinators (or the PAN coordinator). Similarly, the energy consumption of coordinator nodes can be obtained by Equation 52, which consists of 10 parts as in order for backoff, receiving data packet transmission from its children node, transmitting ACK to its children nodes, transmitting CCA, transmitting data packet, receiving ACK from its coordinator (i.e., PAN coordinator), idle, transmitting beacon, receiving beacon, and sleeping, respectively. The energy consumption of the PAN coordinator can be obtained by Equation 53, which consists of five parts as in order for idle, transmitting beacon, receiving data packet, transmitting ACK, and sleeping. Finally, the total energy consumption of a cluster tree network, $E_{\text {total }}$, can be obtained by Equation 54 . 


$$
E_{\text {dev }}=N_{\text {dev }} T_{\text {sim }}\left[\begin{array}{l}
\left(P W_{\text {idle }} \frac{S D_{\text {dev }}}{B I_{\text {PAN }}} \sum_{i=0}^{m} \sum_{j=0}^{w_{i}-1} \sum_{k=0}^{R} b_{i, j, k}\right)+\left(\frac{2 P W_{t x}}{I N T V} \frac{L_{c c a}}{R_{b}} d_{\text {node }} \sum_{i=0}^{m} \sum_{k=0}^{R}\left(b_{i,-1, k}+b_{i,-2, k}\right)\right) \\
+\left(\frac{P W_{t x}}{I N T V} \frac{L_{\text {data }}}{R_{b}} P_{\text {succoord }} d_{\text {node }}\right)+\left(P W_{\text {idle }} P[I D L E] \frac{S D_{\text {dev }}}{B I_{\text {PAN }}}\right) \\
+\frac{P W_{\text {rx }}}{R_{b}} \frac{L_{\text {beacon }}}{B I_{\text {coord }}}+\left(\frac{P W_{r x}}{I N T V} \frac{L_{\text {ack }}}{R_{b}} P_{\text {succoord }}\right)+\left(P W_{\text {sleep }}\left(1-D C_{\text {dev }}\right) \frac{B I_{\text {dev }}}{B I_{\text {PAN }}}\right)
\end{array}\right]
$$

$$
E_{\text {coord }}=N_{\text {coord }} T_{\text {sim }}\left[\begin{array}{l}
\left(N_{\text {ch }} P W_{\text {idle }} \frac{S D_{\text {coord }}}{B I_{P A N}} \sum_{i=0}^{m} \sum_{j=0}^{w_{i}-1} \sum_{k=0}^{R} b_{i, j, k}\right)+\left(N_{\text {ch }} P_{\text {succoord }} \frac{P W_{r x}}{I N T V} \frac{L_{\text {data }}}{R_{b}}\right) \\
+\left(N_{\text {ch }} d_{\text {node }} P_{\text {succoord }} \frac{P W_{t x}}{I N T V} \frac{L_{\text {ack }}}{R_{b}}\right) \\
+\left(\frac{2 P W_{t x}}{I N T V} \frac{L_{\text {cca }}}{R_{b}} P_{\text {succoord }} d_{\text {node }} \sum_{i=0}^{m} \sum_{k=0}^{R}\left(b_{i,-1, k}+b_{i,-2, k}\right)\right) \\
+\left(\frac{P W_{t x}}{I N T V} \frac{L_{\text {data }}}{R_{b}} P_{\text {succoord }} P_{\text {suc PAN }} d_{\text {node }}\right)+\left(\frac{P W_{r x}}{I N T V} \frac{L_{\text {ack }}}{R_{b}} P_{\text {sucPAN }}\right) \\
+\left(P W_{\text {idle }} P[I D L E] \frac{S D_{\text {coord }}}{B I_{P A N}}\right)+\left(P W_{t x} \frac{L_{\text {beacon }}}{R_{b}} \frac{d_{\text {node }}}{B I_{\text {coord }}}\right) \\
+\left(\frac{P W_{r x}}{R_{b}} \frac{L_{\text {beacon }}}{B I_{\text {PAN }}}\right)+\left(P W_{\text {sleep }}\left(1-D C_{\text {coord }}\right) \frac{B I_{\text {coord }}}{B I_{P A N}}\right)
\end{array}\right]
$$

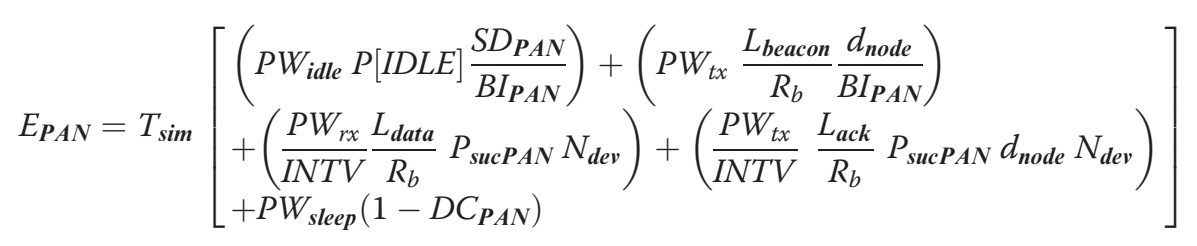$$
E_{\text {total }}=E_{\text {dev }}+E_{\text {coord }}+E_{\boldsymbol{P A N}}
$$

\section{Simulation and analysis results}

In this section, simulation experiments are performed by using the extended network simulator NS-2 to validate the analysis and performance evaluation. We consider a cluster topology with 1 PAN coordinator, 3 coordinator nodes, and 9 device nodes as shown in Figure 3, where the distance between nodes ( $\left.d_{\text {node }}\right)$ is equal to $10 \mathrm{~m}$. The packet arrival rate follows the Poisson distribution with the INTV of data packets from 0.1 to 1 , where packets have the same length of 560 bits. To simulate the performance of power consumption, we consider the radio parameters of Chipcon's CC2420 2.4 GHz for the IEEE 802.15.4 RF transceiver [20], where the transmitting power $P W_{t x}$, the receiving power $P W_{r x}$, the idle power $P W_{\text {idle, }}$, and the sleeping power per time unit $P W_{\text {sleep }}$ are $31.32 \mathrm{~mW}, 35.28 \mathrm{~mW}, 712 \mu \mathrm{W}$, and $144 \mathrm{nW}$, respectively [16]. The $\mathrm{BO}$ and $\mathrm{SO}$ settings follow the proposed SABTS algorithm, but they are fixed for IEEE 802.15.4 standard, i.e., $\mathrm{BO}=\mathrm{SO}=6$. Table 1 summarizes the simulation parameters. We compute the probabilities of collision of packet transmission and entering the next backoff stage in a certain backoff stage. We also compare the analytical (ana) and simulation (sim) results between the proposed algorithm (SABTS) and IEEE 802.15.4 standard (Std) for network goodput, total network energy consumption, and the probability of successful packet transmission arriving at PAN.

Figure 6 shows the probabilities of collision of packet transmission and entering the next backoff stage in a certain backoff stage, denoted as $X$ and $Y$, respectively, against the INTV of data packets by analytical model. The probabilities of $X$ and $Y$ of SABTS are lesser than those of IEEE 802.15.4 standard. Normally, the lesser probabilities of $X$ and $Y$, the higher probability of successful packet transmission, which implies the lesser energy consumption needed for packet transmission, i.e., SABTS should have higher network goodput and lesser energy consumption than those of IEEE 802.15.4 standard.

Figure 7 shows the probability of successful transmission arrives at the PAN coordinator $\left(P_{\text {sucPAN }}\right)$ against the INTV of data packets by analytical and simulation. SABTS obtains higher probability of successful transmission arriving at the PAN coordinator than that of IEEE 
Table 1 The simulation parameters

\begin{tabular}{lc}
\hline Parameter & Value \\
\hline Channel bandwidth & $250 \mathrm{kbps}$ \\
Packet length $\left(L_{\text {data }}\right)$ & 560 bits \\
UBP & $80 \mathrm{bits}$ \\
MAC header & $2 \mathrm{UBP}$ \\
ACK length $\left(L_{\mathrm{ACK}}\right)$ & $88 \mathrm{bits}$ \\
$d_{\text {node }}$ & $10 \mathrm{~m}$ \\
$P W_{\text {tx }}$ & $31.32 \mathrm{~mW}$ \\
$P W_{\text {rx }}$ & $35.28 \mathrm{~mW}$ \\
$P W_{\text {idle }}$ & $712 \mu \mathrm{W}$ \\
$P W_{\text {sleep }}$ & $144 \mathrm{nW}$ \\
$B O=S O(S t d)$. & 6 \\
\hline
\end{tabular}

802.15.4 standard because the length of active period and the beacon transmission time are managed appropriately. The probability of successful transmission of IEEE 802.15.4 standard decreases more as traffic load increases because the heavy collision occurs either between beacons or between beacon and data frame.

Figure 8 shows the network goodput against the INTV of data packets. The network goodput obtained by simulation is very close to that obtained by analysis model. It is obvious the network goodput of SABTS is higher than that of IEEE 802.15.4 standard especially when the traffic load increases. The probability of collisions of transmitting beacons and data packets can be decreased, because the beacon transmission time and active period length are accurately managed by SABTS. In the light traffic load (i.e., INTV is equal to 1 and 0.9), the network goodput of SABTS is almost same as those of IEEE 802.15.4 standard; however, SABTS outperforms IEEE 802.15.4 standard as the traffic load increases.

Figure 9 shows the network energy consumption against the INTV of data packets. SABTS consumes lesser network energy than that of IEEE 802.15.4

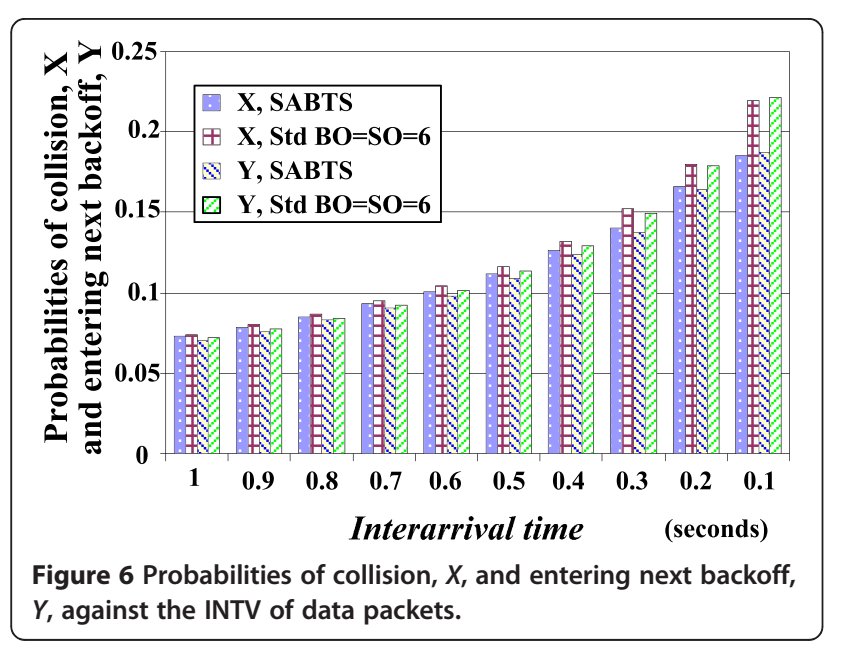

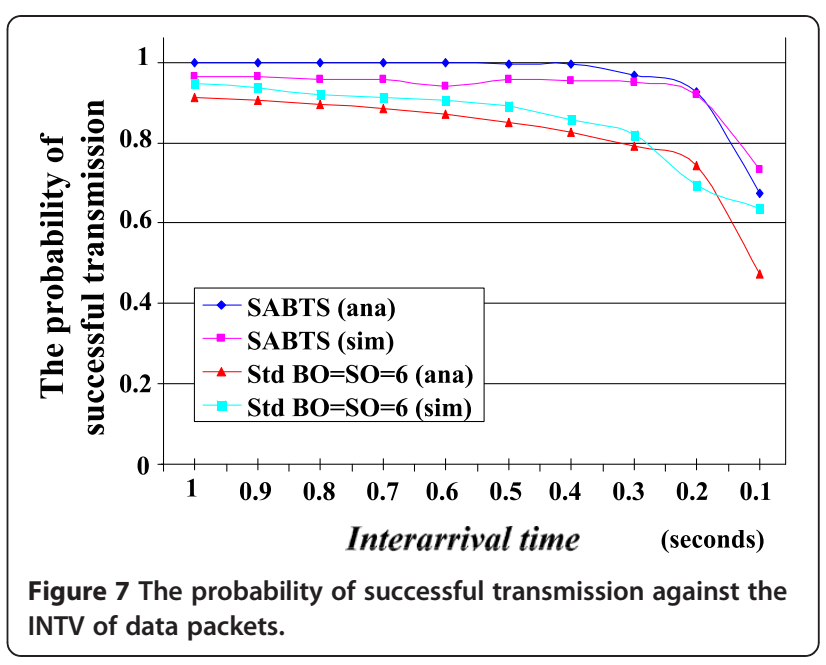

standard. The energy consumption is obtained by summing the energy consumptions of the PAN coordinator, coordinator nodes, and children device nodes. By SABTS, the coordinator nodes and device nodes can save energy consumption during the inactive period as shown in Figure 4. Moreover, SABTS gains the greater probability of successful transmission than that of IEEE 802.15.4 standard especially in heavy traffic load, which means that SABTS minimizes the energy consumption when retransmitting data packets.

\section{Conclusions}

In this article, SABTS has been proposed to adjust two system parameters of superframe (i.e., $\mathrm{BO}$ and $\mathrm{SO}$ ) and set the precise time for beacon transmission to achieve low energy consumption and to alleviate the collision of beacon and data packet transmissions. This article presented a comprehensive Markov chain analysis of IEEE 802.15.4, specifically for cluster tree network, to predict the network goodput as well as the network energy

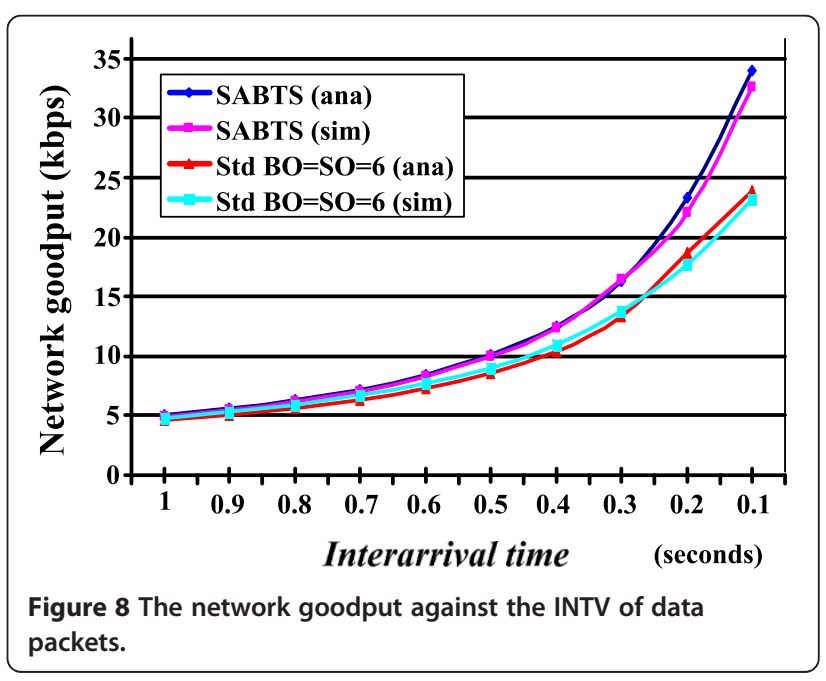




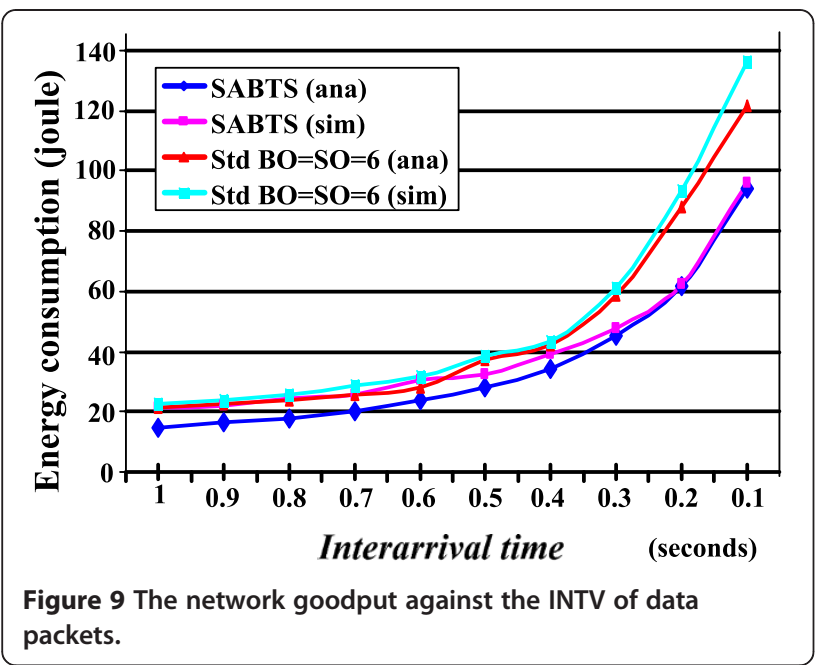

consumption. The validity of the analytical model is shown by closely matching its predictions to the simulation results. The results obtained by analytical model and simulation experiments show that SABTS performs better than IEEE 802.15.4 standard especially in the network goodput and the network energy consumption. However, this article pays much attention to the slotted CSMA/CA channel access mechanism within the CAP of a superframe, but for certain applications with low latency or specific bandwidth requirements it needs dedicated guaranteed time slots (GTS) channel to transmit its data packets without contention. For further research, the SABTS algorithm will be expected to consider GTS within the CFP of a superframe to improve the network performance.

\section{Abbreviations}

ACK: Acknowledgement; BE: Backoff exponent; BI: Beacon interval; BO: Beacon order; CAP: Contention access period; CCA: Clear channel assessment; CFP: Contention free period; CSMA/CA: Carrier sense multiple access with collision avoidance; CW: Contention window; IFS: Interframe space; INTV: Interarrival time of data packets; LR-WPAN: Low-rate wireless personal area network; MAC: Medium access control sublayer; NB: Number of backoffs; PAN: Personal area network; SD: Superframe duration (in symbols); SDT: Superframe duration time (in seconds); SO: Superframe order; UBP: Unit backoff period (80 bits).

\section{Competing interests}

The authors declare that they have no competing interests.

\section{Acknowledgments}

This study was supported in part by the National Science Council (NSC) of Taiwan under Grant No. NSC 96-2221-E-011-055.

\section{Author details}

${ }^{1}$ National Taiwan University of Science and Technology, 43, Keelung Rd. Section 4, Taipei 106, Taiwan. ${ }^{2}$ Ling Tung University, 1, Ling Tung Rd., Taichung 408, Taiwan.

Received: 24 March 2012 Accepted: 4 June 2012

Published: 17 July 2012

\section{References}

1. IEEE 802.15.4, Part 15.4, Wireless Medium Access Control (MAC) and Physical Layer (PHY) Specifications for Low-Rate Wireless Personal Area Networks (WPANs) (IEEE standard for information technology, 2006)

2. A Koubaa, A Cunha, M Alves, E Tovar, TDBS: a time division beacon scheduling mechanism for ZigBee cluster-tree wireless sensor networks. Real-Time Syst. J. 40(3), 321-354 (2008)

3. J-W Kim, J Kim, D-S Eom, Multi-dimensional channel management scheme to avoid beacon collision in LR-WPAN. IEEE Trans. Consum. Electron. 54(2), 396-404 (2008)

4. J Lu, A Van Den Bossche, E Campo, A new beacon scheduling mechanism for mesh wireless personal area networks based on IEEE 802.15.4, in IEEE 16th Conference on Emerging Technologies \& Factory Automation (ETFA), (Toulouse, France, 2011), pp. 1-4

5. TR Park, TH Kim, JY Choi, S Choi, WH Kwon, Throughput and energy consumption analysis of IEEE 802.15.4 slotted CSMA/CA. IEEE. Electron. Lett. 41(18), 1017-1019 (2005)

6. T-J Lee, HR Lee, MY Chung, MAC throughput limit analysis of slotted CSMA CA in IEEE 802.15.4 WPAN. IEEE Commun. Lett. 10(7), 561-563 (2006)

7. S Pollin, M Ergen, S Ergen, B Bougard, L Der Perre, I Moerman, A Bahai, $P$ Varaiya, F Catthoor, Performance analysis of slotted carrier sense IEEE 802.15.4 medium access layer. IEEE Trans. Wirel. Commun. 7(9), 3359-3371 (2008)

8. Y Zhang, F Shu, Packet size optimization for goodput and energy efficiency enhancement in slotted IEEE 802.15.4 networks, in IEEE Wireless Communications and Networking Conference, (Budapest, Hungary, 2009), pp. 1-6

9. J He, Z Tang, H-H Chen, Q Zhang, An accurate and scalable analytical model for IEEE 802.15.4 slotted CSMA/CA networks. IEEE Trans. Wirel. Commun. 8(1), 440-448 (2009)

10. Z Xiao, C He, L Jiang, An analytical model for IEEE 802.15.4 with sleep mode based on time-varying queue, in IEEE International Conference on Communications (ICC), (Kyoto, Japan, 2011)

11. C Buratti, Performance analysis of IEEE 802.15.4 beacon-enabled mode. IEEE Trans. Veh. Technol. 59, 2031-2045 (2010)

12. Z Tao, S Panwar, D Gu, J Zhang, Performance analysis and a proposed improvement for the IEEE 802.15.4 contention access period, vol. 4, in IEEE Wireless Communications and Networking Conference, (Las Vegas, USA, 2006), pp. 1811-1818

13. P Park, P Di Marco, P Soldati, C Fischione, KH Johansson, A generalized Markov chain model for effective analysis of slotted IEEE 802.15.4, in IEEE 6th International Conference on Mobile Adhoc and Sensor Systems, (Macau, China, 2009), pp. 130-139

14. Y-K Huang, A-C Pang, H-N Hung, A comprehensive analysis of low-power operation for beacon-enabled IEEE 802.15.4 wireless networks. IEEE Trans. Wirel. Commun. 8(11), 5601-5611 (2009)

15. M Khanafer, M Guennoun, HT Mouftah, Adaptive sleeping periods in IEEE 802.15.4 for efficient energy savings: markov-based theoretical analysis, in IEEE International Conference on Communications (ICC), (Kyoto, Japan, 2011)

16. B Gao, C He, L Jiang, Modeling and analysis of IEEE 802.15.4 CSMA/CA with sleep mode enabled, in International Conference on Communication Systems, (Guangzhou, China, 2008), pp. 6-11

17. CY Jung, HY Hwang, DK Sung, GU Hwang, Enhanced Markov chain model and throughput analysis of the slotted CSMA/CA for IEEE 802.15.4 under unsaturated traffic conditions. IEEE Trans. Veh. Technol. 58(1), 473-478 (2009)

18. B Shrestha, E Hossain, S Camorlinga, A Markov model for IEEE 802.15.4 MAC with GTS transmissions and heterogeneous traffic in non-saturation mode, in IEEE International Conference on Communication Systems (ICCS), (Singapore, 2010), pp. 56-61

19. M Martalo, S Busanelli, G Ferrari, Markov chain-based performance analysis of multihop IEEE 802.15.4 wireless networks. Performance Eval. J. 66, 722-741 (2009)

20. AS Chipcon, SmartRF ${ }^{\oplus}$ CC2420 datasheet (rev 1.2) (Chipcon Corp, 2004)

doi:10.1186/1687-1499-2012-219

Cite this article as: Lee et al: Analysis of superframe adjustment and beacon transmission for IEEE 802.15.4 cluster tree networks. EURASIP Journal on Wireless Communications and Networking 2012 2012:219. 